\title{
Communication
}

\section{Fruit Morphology Measurements of Jujube Cultivar 'Lingwu Changzao' (Ziziphus jujuba Mill. cv. Lingwuchangzao) during Fruit Development}

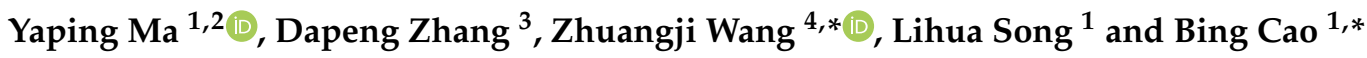 \\ 1 School of Agriculture, Ningxia University, Yinchuan 750021, China; YapingMa@njfu.edu.cn (Y.M.); \\ slh382@126.com (L.S.) \\ 2 College of Forestry, Nanjing Forestry University, Nanjing 210037, China \\ 3 USDA-ARS, Sustainable Perennial Crops Laboratory, Beltsville, MD 20705, USA; dapeng.zhang@usda.gov \\ 4 Department of Plant Science and Landscape Architecture, University of Maryland, \\ College Park, MD 20742, USA \\ * Correspondence: zwang134@umd.edu (Z.W.); bingcao2006@126.com (B.C.)
}

check for updates

Citation: Ma, Y.; Zhang, D.; Wang, Z.; Song, L.; Cao, B. Fruit Morphology Measurements of Jujube Cultivar 'Lingwu Changzao' (Ziziphus jujuba Mill. cv. Lingwuchangzao) during Fruit Development. Horticulturae 2021, 7, 26. https://doi.org/10.3390/ horticulturae7020026

Academic Editor: Esmaeil Fallahi

Received: 12 January 2021

Accepted: 4 February 2021

Published: 6 February 2021

Publisher's Note: MDPI stays neutral with regard to jurisdictional claims in published maps and institutional affiliations.

Copyright: (c) 2021 by the authors. Licensee MDPI, Basel, Switzerland. This article is an open access article distributed under the terms and conditions of the Creative Commons Attribution (CC BY) license (https:/ / creativecommons.org/licenses/by/ $4.0 /)$.

\begin{abstract}
Lingwu Changzao' (Ziziphus jujuba Mill. cv. Lingwuchangzao), a cultivar of Ziziphus in the Rhamnaceae family, is a traditional jujube cultivar in Ningxia, China. For 'Lingwu Changzao', morphological traits are prominent in characterizing fruit yield, quality, and consumer acceptance. However, morphological measurements for 'Lingwu Changzao' cultivation are limited. Therefore, the objective of this study is to measure the growing patterns of selected morphological traits during 'Lingwu Changzao' fruit development. Eight morphological traits, including four fruit traits (fruit length, diameter, weight, and flesh (mesocarp) thickness), three stone traits (stone length, diameter, and weight), and fruit firmness (also known as fruit hardness), were measured over a 3-mo (months) period, covering a completed fruit development period. Results indicate that the growing patterns of fruit traits coincide with double ' $S$ ' growth curves, which mainly present the growth of 'Lingwu Changzao' fruit. Increases of stone traits terminated in the early fruit growth period, while fruit traits continuously increased till the end of the 3-mo period. That implies a high fruit-stone ratio, i.e., a desirable quality attribute for 'Lingwu Changzao' as fresh-eating fruits. The results presented in this study can serve as one part of the standard dataset for jujube fruit cultivation in China, and it can also support decisions in plant breeding and field managements for 'Lingwu Changzao'.
\end{abstract}

Keywords: 'Lingwu Changzao'; fruit morphology; stone morphology

\section{Introduction}

Chinese Jujube (jujube, red date, Chinese date) belongs to the Ziziphus species in the Rhamnaceae family. It is native to China and has been cultivated for 700-800 years [1,2]. Chinese Jujube has outstanding adaptability, nutritious fruits, and multi-purpose utilization in food and traditional medicine, and it is an ideal economic crop in arid and semiarid regions under temperate and subtropical climate. The annual production of jujube in China is $>1.1 \times 10^{10} \mathrm{~kg}$, supporting a market value of over $\$ 14$ billion [1].

Jujube cultivars are typically classified according to the purpose of consumption and utilization. Some are cultivated for fresh eating, some are good for drying and food processing. 'Lingwu Changzao' (Ziziphus jujuba Mill. cv. Lingwuchangzao) is a high quality fresh-eating cultivar in Ningxia, China, featured for its superior taste, texture, and high fruit/stone ratio. The annual production of 'Lingwu Changzao' reached $\sim 1.8 \times 10^{7} \mathrm{~kg}$, with a total cultivation area of $\sim 4.5 \times 10^{7} \mathrm{~m}^{2}$ in $2017[3,4]$.

With an expansion of the fresh-eating jujube market and an increasing consumer demand for fruit quality and appearance, it is imperative to select jujube cultivars with desirable fruit morphology. Fruit morphological traits include fruit color, fruit size, fruit 
shape, flesh (mesocarp) weight, and stone weight. Remarkable variations of fruit morphological traits have been reported in a range of jujube cultivars [5]. Quantitative traits, such as fruit size and fruit weight are yield components, whereas other traits, such as skin color, fruit shape, fruit firmness (also known as fruit hardness), and fruit/stone ratio, are quality attributes. Since some quality attributes can be expressed with quantitative traits (e.g., fruit shape is defined by the ratio of fruit length and diameter, fruit/stone ratio is computed with fruit flesh weight and stone weight), quantitative morphological traits become critical in demarcating fruit yield and fruit quality.

Measurements of quantitative morphological traits are relatively easy. Observing the changes of quantitative traits during a completed jujube fruit developing period not only enhances our understanding of the fruit growth patterns, but also provides references for field management in improving fruit yield and quality. For example, the ideal time for fertilization and irrigation could be the flesh growth period rather than stone growth period. A standard dataset that presents the jujube fruit development under a variety of ambient conditions, such as weather or field managements, can also support decisions in plant breeding. However, for under-investigated fruit tree crops, such as 'Lingwu Changzao', dataset for fruit and stone morphological traits have been rarely reported. Therefore, a study of 'Lingwu Changzao' fruit morphology, with multiple fruit and stone traits over a completed fruit growth period, is necessary.

The objectives of this study are to measure the growing patterns of eight selected fruit morphological traits during jujube fruit development, including four fruit traits (fruit length, fruit diameter, fruit weight, and fruit flesh (mesocarp) thickness), three stone traits (stone length, stone diameter, and stone weight), and fruit firmness.

\section{Materials and Methods}

'Lingwu Changzao' fruit trees were planted in an orchard of the Ningxia University Horticultural Research Station, located in Yongning County, Ningxia, China $\left(38.78^{\circ} \mathrm{N}\right.$, $106.07^{\circ} \mathrm{E}, 1117 \mathrm{~m}$ in altitude). The experiment site was of arid continental climate, with an annual frost-free period of 140-160 d. The annual average sunshine was $3000 \mathrm{~h}$, and the average temperature and precipitation were $8.5^{\circ} \mathrm{C}$ and $180-300 \mathrm{~mm}[6]$. The orchard was of $3300 \mathrm{~m}^{2}$. Fruit trees in the orchard were 4-yr-old (Figure 1a), with an average height of $1.7 \mathrm{~m}$. Each tree occupied an area of $2 \times 3 \mathrm{~m}^{2}$. Thirty healthy fruit trees were randomly selected for this study.

Eight measurements were performed from early fruiting (12 July 2017) to fruit ripening (4 October 2017), and consecutive measurements were separated by a 12-d interval. For each measurement, 10 fruit samples from one single fruit tree were hand-harvested. Figure 1c presents the photographs of the jujube fruits on the sampling dates. Fruit length, fruit diameter, flesh thickness, stone length, and stone diameter were determined with a digital caliper of $0.01 \mathrm{~mm}$ resolution (Figure 1b). Fruit weight and stone weight were obtained with an electronic scale. Fruit firmness $\left(\mathrm{kg} \mathrm{cm}^{-2}\right)$ of each jujube fruit was the average value of two measurements at opposite sides near the fruit equatorial zone. A GY-4 digital fruit penetrometer (Zhejiang Top Instrument Co. Ltd., Hangzhou, China) with a $3 \mathrm{~mm}$ diameter probe was used to determine the firmness values, and a small piece of skin was removed from the fruit surface before firmness measurements. Stone traits were determined after the fruit flesh was completely removed.

Observed results were analyzed with Tukey's HSD multiple comparisons, performed at a significance level of 0.01 . Furthermore, Pearson analysis was applied to identify the correlation among the measured morphological traits and investigate the relations between morphological traits and yield. 


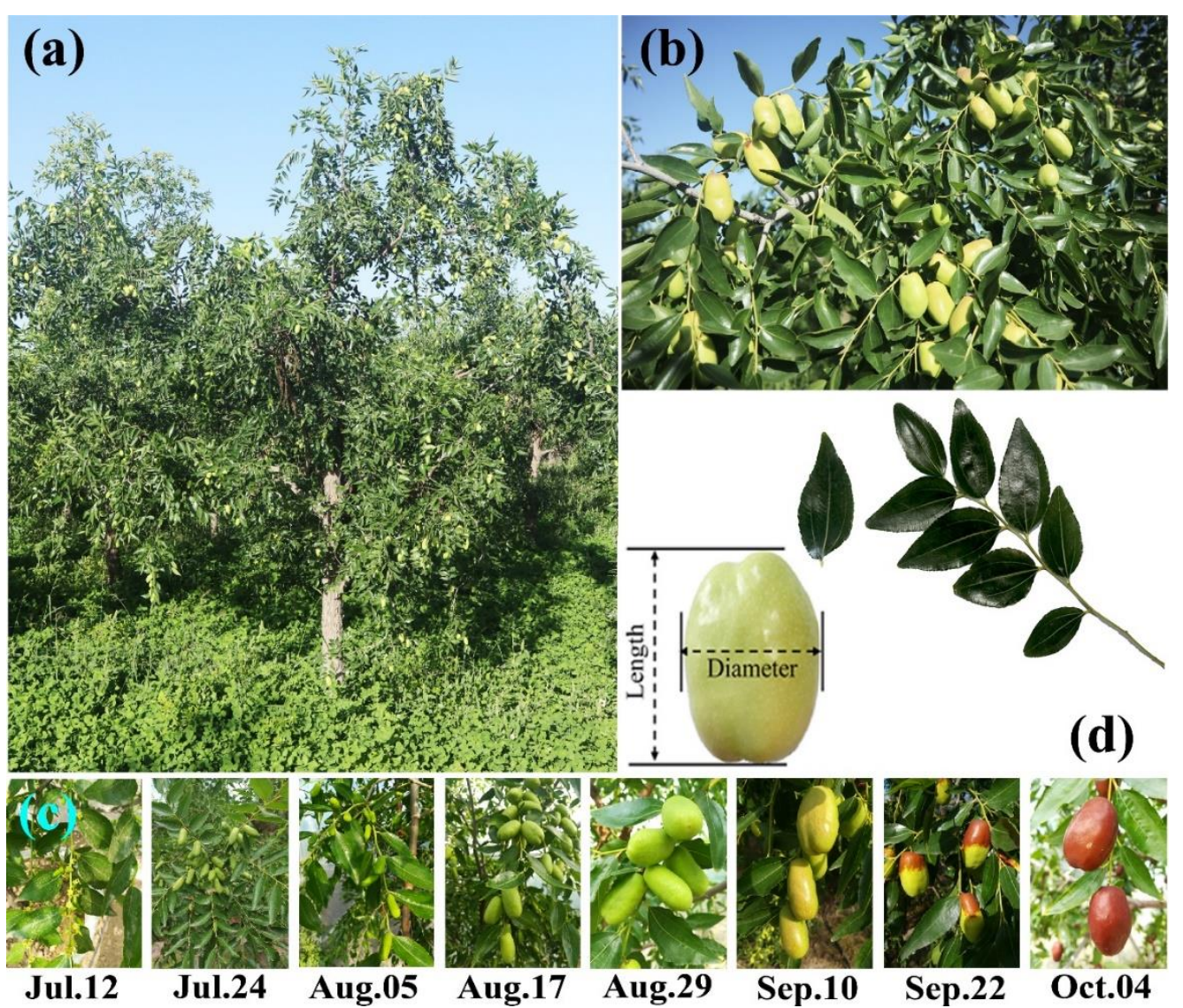

Figure 1. The adult tree of 'Lingwu Changzao' (a), fruit and leaves (b), fruits on the sampling dates (c), and an illustrative measurement for fruit length and diameter (d).

\section{Results and Discussion}

Box plots in Figure 2 represent the growing patterns of the selected morphological traits during the experiment period. Within each box, the center red line represents the median value, while the red diamond represents the mean value. The upper and lower edges of a given box present the $25 \%$ and $75 \%$ percentiles, while the whiskers extend to the most extreme data points that are not considered as outliers, i.e., $5 \%$ and $95 \%$ percentiles. Box plots also imply data normality, i.e., medians and means are similar, and upper and lower box edges, as well as whiskers are nearly symmetric about the means. The green dash lines present the fitted Logistic growth curves, except for the fruit firmness, where a smoothing spline is applied to show its "blow-off top" pattern. The Tukey's HSD multiple comparison results for each trait are marked with blue letters (Figure 2). Figure 3 presents the Pearson correlation analysis among the selected morphological traits at a significance level of 0.01. The correlation coefficients are shown in Figure 3 and represented with dot-size and dot-color. 

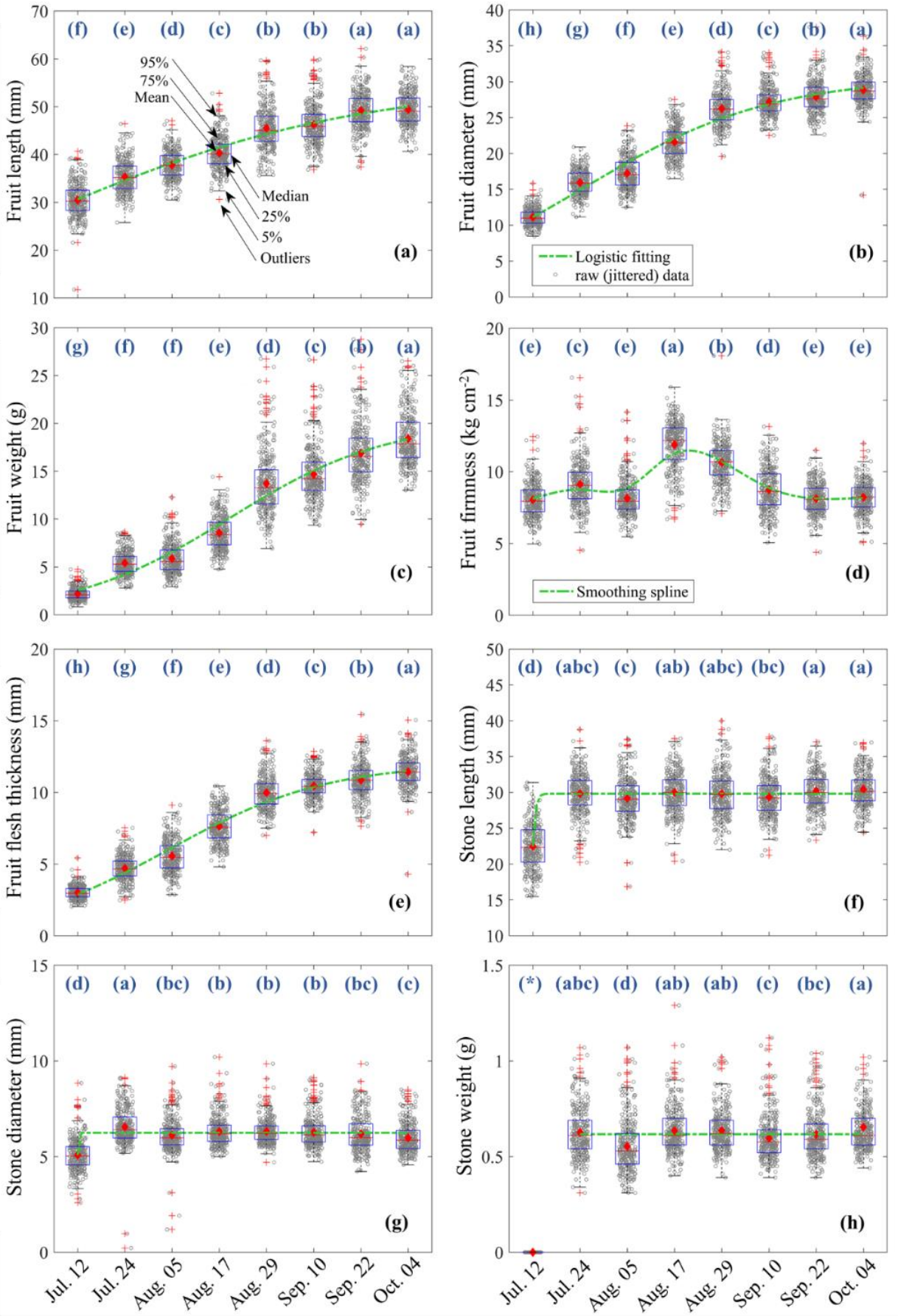

Figure 2. Box plots the measuring results of the 8 selected traits of 'Lingwu Changzao' fruit, including (a) fruit length, (b) fruit diameter, (c) fruit weight, (d) fruit firmness, (e) fruit flesh thickness, (f) stone length, (g) stone diameter, and (h) stone weight. Logistic growth curves or Smoothing-spline regression curves are presented with dashed green lines. The blue characters indicate multiple comparison results. ${ }^{*}$ Stone weight measurements cannot be performed on $12 \mathrm{July}$, because the seed coat tissue was not formed, such that the complete fruit stones cannot be obtained. 


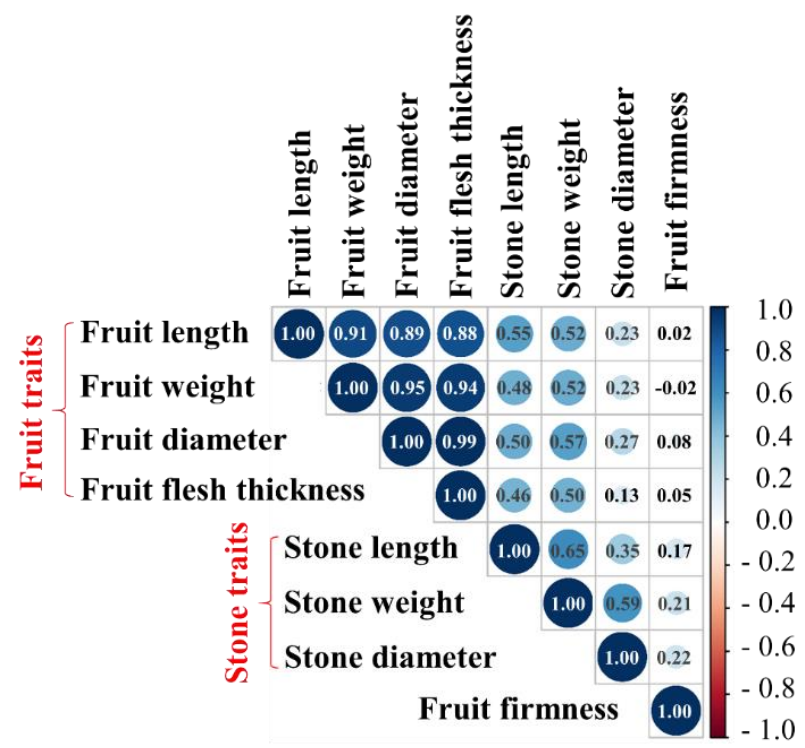

Figure 3. Pearson correlation analysis of the eight traits of 'Lingwu Changzao' fruit. The correlation coefficients are included in the figure.

The growth of selected traits followed three patterns. Four fruit traits increased with respect to time, coinciding with a double ' $S$ ' growth curve reported in $[7,8]$. The growth rates were maintained relatively large during the whole experiment period; therefore, in general, values of the fruit traits in two consecutive measurements showed significant differences. Fruit diameter, fruit weight, and fruit flesh thickness had continuously increasing patterns, especially near the end of the experiment period. However, for fruit length, no significant differences were shown between 29 August and 10 September and between 22 September and 4 October. That implied the existence of a turning point, where the increases of fruit length gradually terminated, while the increases of fruit diameter, fruit weight and fruit flesh thickness continued. The differences between fruit length and the other three fruit traits can also be observed in Figure 3, where the correlation between fruit length and fruit diameter, fruit weight or fruit flesh thickness are relatively weak $(\sim 0.9)$ comparing to the correlation coefficients among fruit diameter, fruit weight and fruit flesh thickness ( 0.95). That also explained the fact reported in [3], where comparing to fruit length, fruit diameter could be an appropriate feature to represent the fruit yield. Because of the importance of fruit traits in yields, the fitted Logistic growth curves for the four selected fruit traits are summarized in Table 1, using the toolbox proposed by [9].

Table 1. The fitted logistic growth curves for fruit traits (assume time, $t$, has unit "d" and $t=0$ on 12 July).

\begin{tabular}{cccc}
\hline Fruit Traits & Logistic Growth Curves & $\begin{array}{c}p \text {-Value (via Analysis } \\
\text { of Variance) }\end{array}$ & $\begin{array}{c}\text { MSE (Degrees of } \\
\text { Freedom, df = 2397) }\end{array}$ \\
\hline $\begin{array}{c}\text { Fruit length } \\
(\mathrm{mm})\end{array}$ & $y=\frac{41.84}{1+\left(\frac{41.84-18.95}{18.95}\right) e^{-0.03 t}}$ & $<0.0001$ & 3.62 \\
$\begin{array}{c}\text { Fruit diameter } \\
(\mathrm{mm})\end{array}$ & $y=\frac{20.87}{1+\left(\frac{20.87-3.30}{3.30}\right) e^{-0.064 t}}$ & $<0.0001$ & 2.15 \\
$\begin{array}{c}\text { Fruit weight } \\
(\mathrm{g})\end{array}$ & $y=\frac{18.90}{1+\left(\frac{18.90-1.90}{1.90}\right) e^{-0.056 t}}$ & $<0.0001$ & 2.39 \\
$\begin{array}{c}\text { Fruit flesh thickness } \\
(\mathrm{mm})\end{array}$ & $y=\frac{9.53}{1+\left(\frac{9.53-1.11}{1.11}\right) e^{-0.069 t}}$ & $<0.0001$ & 1.03 \\
\hline
\end{tabular}

During the early experiment period, stone traits presented a steep increasing growing pattern, and then maintained constant (Figure $2 \mathrm{f}-\mathrm{h}$ ). Although some significant differences were shown for stone traits near the end of the experiment period, the absolute differences among the measured values were essentially small. Stones usually account for $10 \sim 15 \%$ 
of the total fruit weight and somehow influence fruit length [10]. However, for 'Lingwu Changzao', stone growth nearly stopped in the early experiment period, such that stone length occupied $\sim 60 \%$ of fruit length, while stone weight was only $\sim 6 \%$ of fruit weight. Hence, 'Lingwu Changzao' had a relatively high fruit/stone ratio [11-13].

Fruit firmness is presented in Figure $2 \mathrm{~d}$. The values were roughly constant at the beginning, then increased to the maximum on 17 August; after that, fruit firmness values gradually decreased to a stable value. Such a pattern was different from any other morphological traits considered in this study. Hence, the correlation coefficients between fruit firmness and other traits achieved relatively small values (Figure 3).

\section{Summary}

In this study, morphological traits of jujube cultivar 'Lingwu Changzao' (Ziziphus jujuba Mill. cv. Lingwuchangzao) were measured over a completed fruit developing period. Four fruit traits (fruit length, diameter, weight, and flesh thickness) presented relatively stable growing patterns during the experiment period; the growing speed of three stone traits (stone length, diameter, and weight) was initially large, but reduced to nearly zero later; the fruit firmness (also known as fruit hardness) increased from constant trend to a maximal value and then decreased. Pearson correlation analysis indicated strong correlations among fruit diameter, flesh thickness, and fruit weight. In conclusion, fruit diameter and fruit flesh thickness are main contributors to fruit yield; with early termination of stone growth, the biological basis of the high fruit-stone ratio in 'Lingwu Changzao' is proved. Future research should include the studies to dissect and quantify genotype effects, while multi-year experiments should be performed to identify the responses of fruit morphology to a variety of weather conditions and complete a standard dataset to support jujube cultivation in China.

Author Contributions: B.C. and L.S. designed the research; Y.M. conducted the experiment, analyzed data, and drafted the manuscript; Z.W. supervised data analysis and revised the manuscript; D.Z. provided suggestions for the experiments and the revised manuscript. All authors approved the final manuscript. All authors have read and agreed to the published version of the manuscript.

Funding: This research was supported by Key research and development project of Ningxia (Grant No. 2018BFH03015), and Science and Technology Innovation Project of first-class universities Construction in western China for Ningxia University (Grant No. ZKZD2017008).

Institutional Review Board Statement: Not applicable.

Informed Consent Statement: Not applicable.

Data Availability Statement: The authors are pleased to share the data upon request.

Conflicts of Interest: The authors report no conflict of interest.

\section{References}

1. Liu, W.R.; Jiang, B.; Peng, Q.W.; He, X.M.; Lin, Y.E.; Wang, M.; Liang, Z.J.; Xie, D.S.; Hu, K.L. Genetic analysis and QTL mapping of fruit-related traits in wax gourd (Benincasa hispida). Euphytica 2018, 214, 136. [CrossRef]

2. Wang, B.; Huang, Q.; Venkitasamy, C.; Chai, H.K.; Gao, H.; Cao, W.; Lv, X.G.; Pan, Z.L. Changes in phenolic compounds and their antioxidant activity in jujube (Ziziphus jujuba Mill.) during three edible maturity stages. LWT Food Sci. Technol. 2016, 66, 56-62. [CrossRef]

3. Ma, Y.P.; Cao, B.; Li, Z.W.; Su, W.D. Fruit characters and their correlations of ziziphus jujuba 'Lingwu Changzao'. Chin. Agric. Sci. Bull. 2017, 33, 92-96.

4. Li, J.W.; Fan, L.P.; Ding, S.D.; Ding, X.L. Nutritional composition of five cultivars of Chinese jujube. Food Chem. 2007, 103, 454-460. [CrossRef]

5. Ivanišová, E.; Grygorieva, O.; Abrahamová, V.; Schubertova, Z.; Terentjeva, M.; Brindza, J. Characterization of morphological parameters and biological activity of jujube fruit (Ziziphus jujuba Mill.). J. Berry Res. 2017, 7, 249-260. [CrossRef]

6. Song, L.H.; Cao, B. Effect of cover-cultivation on soil temperature and growth of Ziziphus jujuba Mill. 'Lingwu Changzao'. Acta Hortic. 2016, 1116, 89-92. [CrossRef]

7. Chen, Y.P.; Zhang, Y.C.; Jing, H.X.; Su, W.D. The characteristic of photosynthesis and the growth of fruit of zizyphus jujube Mill cv. Lingwuchangzao. North. Hortic. 2013, 19, 1-4. 
8. Jing, H.X.; Zhang, Y.C. Structure characteristic of development fruit of ziziphus jujuba cv. Lingwuchagnzao. Guihaia 2014, 34, 565-569. [CrossRef]

9. Sprouffske, K.; Wagner, A. Growthcurver: An R package for obtaining interpretable metrics from microbial growth curves. BMC Bioinform. 2016, 17, 172-175. [CrossRef] [PubMed]

10. Ghnimi, S.; Al-Shibli, M.; Al-Yammahi, H.R.; Al-Dhaheri, A.; Al-Jaberi, F.; Jobe, B.; Kamal-Eldin, A. Reducing sugars, organic acids, size, color, and texture of 21 Emirati date fruit varieties (Phoenix dactylifera, L.). NFS J. 2018, 12, 1-10. [CrossRef]

11. Petruccelli, R.; Ganino, T.; Ciaccheri, L.; Maselli, F.; Mariotti, P. Phenotypic diversity of traditional cherry accessions present in the Tuscan region. Sci. Hort. 2013, 150, 334-347. [CrossRef]

12. Ganopoulos, I.; Moysiadis, T.; Xanthopoulou, A.; Ganopoulou, M.; Avramidou, E.; Aravanopoulos, F.A.; Tani, E.; Madesis, P.; Tsaftaris, A.; Kazantzis, K. Diversity of morpho-physiological traits in worldwide sweet cherry cultivars of GeneBank collection using multivariate analysis. Sci. Hort. 2015, 197, 381-391. [CrossRef]

13. Pouyesh, A.; Lotfi, M.; Ramshini, H.; Karami, E.; Shamsitabar, A.; Armiyoun, E. Genetic analysis of yield and fruit traits in cantaloupe cultivars. Plant Breed. 2017, 136, 569-577. [CrossRef] 